\title{
Obliquity, precession rate, and nutation coefficients for a set of 100 asteroids ${ }^{\star}$
}

\author{
C. Lhotka ${ }^{1}$, J. Souchay ${ }^{2}$, and A. Shahsavari ${ }^{2}$ \\ 1 Department of Mathematics, University of Rome Tor Vergata, 00133 Rome, Italy \\ 2 Observatoire de Paris, SYRTE/UMR-8630 CNRS, 75014 Paris, France \\ e-mail: Jean.Souchay@obspm.fr
}

Received 31 January 2013 / Accepted 18 April 2013

\begin{abstract}
Context. Thanks to various space missions and the progress of ground-based observational techniques, the knowledge of asteroids has considerably increased in the recent years.

Aims. Due to this increasing database that accompanies this evolution, we compute for a set of 100 asteroids their rotational parameters: the moments of inertia along the principal axes of the object, the obliquity of the axis of rotation with respect to the orbital plane, the precession rates, and the nutation coefficients.

Methods. We select 100 asteroids for which the parameters for the study are well-known from observations or space missions. For each asteroid, we determine the moments of inertia, assuming an ellipsoidal shape. We calculate their obliquity from their orbit (instead of the ecliptic) and the orientation of the spin-pole. Finally, we calculate the precession rates and the largest nutation components. The number of asteroids concerned leads to some statistical studies of the output.

Results. We provide a table of rotational parameters for our set of asteroids. The table includes the obliquity, their axes ratio, their dynamical ellipticity $H_{\mathrm{d}}$, and the scaling factor $K$. We compute the precession rate $\dot{\psi}$ and the leading nutation coefficients $\Delta \psi$ and $\Delta \varepsilon$. We observe similar characteristics, as observed by previous authors that is, a significantly larger number of asteroids rotates in the prograde mode $(\approx 60 \%)$ than in the retrograde one with a bimodal distribution. In particular, there is a deficiency of objects with a polar axis close to the orbit. The precession rates have a mean absolute value of $18^{\prime \prime} / y$, and the leading nutation coefficients have an average absolute amplitude of $5.7^{\prime \prime}$ for $\Delta \psi$ and 5.2" for $\Delta \varepsilon$. At last, we identify and characterize some cases with large precession rates, as seen in 25143 Itokawa, with has a precession rate of about $-475^{\prime \prime} / y$.
\end{abstract}

Key words. minor planets, asteroids: general - catalogs - methods: data analysis

\section{Introduction}

The general knowledge of asteroids has been subject to tremendous progress in recent years thanks to very up-to-date groundbased observational techniques and to a significantly increasing number of space missions devoted to close approaches and exploration of these objects. In contrast to orbital parameters, which can generally be deduced precisely from a small set of positions and velocities that are recorded at a suitable interval of time, rotational characteristics of an asteroid necessitates highfrequency measurements. Most studies of these characteristics are based mainly on lightcurve profiles from which rotation rates and global shapes can be extracted. Lightcurve observations are generally not expensive in terms of equipment needed but require a lot of observing time to gather enough data for a precise solution of the spin vector.

In parallel to rotation rates and spin vector orientation, an estimation of the diameter of the asteroids can be obtained in both magnitude and distance determinations. When these results are collected for a large set of objects, statistical studies can be carried out on the distribution of rotation rates and diameters or on the distribution of the orientation of the spin vector with respect to the ecliptic (Pravec et al. 2002). Nevertheless, we note a lack

* Tables 1 and 2 are only available at the CDS via anonymous ftp to cdsarc.u-strasbg.fr (130.79.128.5) or via http://cdsarc.u-strasbg.fr/viz-bin/qcat?]/A+A/556/A8 of information concerning some parameters, when investigating the general data available for asteroids.

First, the estimation of the obliquity is generally not provided, and this is quite surprising, because obliquity is supposed to play a significant role in instances, such as resurfacing and space weathering. Indeed, obliquity directly determines the lengths and the contrasts of the seasons, which exist on the asteroids, and on the terrestrial planets, where these factors are fundamental. A large value of obliquity of an asteroid assumes that some parts of its surface are much more exposed to sunlight than others and that a climatic contrast exists, which is characterized by large differences in solar flux and solar wind exposure. In contrast, a value of obliquity close to zero implies that the solar influence should be the same for each part of the asteroid. These remarks are all available along the asteroidal year, which lasts a few terrestrial years for the main belt asteroids (MBAs).

Second, the precession rates and nutation coefficients of the asteroids are generally not provided by a modern database. The rates and coefficients should be calculated when considering the asteroids in a short-axis rotational state, which is generally confirmed by the observations. Indeed, it was demonstrated theoretically that in the presence of dissipation the equilibrium of a body in rotation is reached when it rotates around its short-axis, which corresponds to the axis with a maximum moment of inertia (Kinoshita 1992), although long-axis mode rotations can possibly be detected around tumbling objects (Burns \& Safronov 1973; Harris 1994). More precisely, the 
energy-dissipation profile may be complex (Pravec et al. 2002), but a reasonable estimate on the timescale $\tau$ of damping of the excited rotation to the lowest state of short-axis rotation ,assuming a low amplitude libration, has been derived by (Burns \& Safronov 1973):

$$
\tau \approx \frac{\mu Q}{\rho K_{3}^{2} R^{2} \omega^{3}},
$$

where $\mu$ is the rigidity of the material composition of the asteroid, $Q$ is the quality factor, which expresses the ratio of the energy contained in the oscillation to the energy lost per cycle, $\rho$ is the bulk density of the body, and $K_{3}^{2}$ is a dimensionless factor that is related to the shape of the body (with a value close to 0.01 for a quasi-spherical body to a value close to 0.1 for a highly elongated one). $R$ is the mean radius of the asteroid, and $\omega$ its angular velocity of rotation. An alternative formula for $\tau$ was given by Harris (1994), who estimated the parameters in (1):

$$
\tau=\frac{P^{3}}{C^{3} D^{2}}
$$

where $P=\frac{2 \pi}{\omega}$ is the rotation period, $D$ is the mean diameter, and $C$ is a constant close to 17 (with a factor $\approx 2.5$ uncertainty). The parameter $\tau$ is expressed here in billion of years, when $P$ is in hours, and $D$ in $\mathrm{km}$. Applying this law, it can be found that the damping timescale is much shorter than the characteristic timescale of events, causing excitation of their rotations as impacts or close encounters (Pravec et al. 2002).

As a consequence, the great majority of asteroids are rotating in a short-axis mode, which means that the axis of rotation should coincide with the axis of figure, which can be defined as the axis with the maximum moment of inertia. As for the Earth, the axis of figure of an asteroid (or its axis of rotation) is therefore supposed to exhibit a slow motion in space, known under the terminology, precession of equinoxes. The precession of equinoxes is because of the torque exerted principally by the Sun and comes from the fact that the asteroid is flattened at its equator. This motion is still verified when the asteroid has a wellpronounced triaxial shape, and when it is far from satisfying the conditions of axi-symmetry of the figure axis. In addition to the precession that is characterized by a conical loop with respect to the inertial space with the obliquity as an aperture angle and that is described in a timescale typically of several tens thousands of years, any asteroid should undergo small nutation oscillations that come from the gravitational torque exerted by the Sun on the flattened body.

Few studies of the combined precession-nutation motion of an asteroid have been carried out until now. For accurate modeling, we can mention Souchay et al. $(2003 \mathrm{~b}, \mathrm{a})$ for the asteroid 433 Eros, which follows the NEAR space mission and Rambaux et al. (2011) for 1 Ceres, the largest body of the asteroid belt. In this last and recent paper, the authors explain that owing to the small amplitudes of the nutation and the very long period of the precession motion, the measurements of the rotational variations of Ceres should be challenging to obtain by observational data as in the instance of the Dawn mission. They also estimated the timescale for Ceres orientation to relax to a generalized Cassini state, finding that the tidal dissipation within this asteroid has probably been too small to drive any significant damping of its obliquity since its formation. Nevertheless, it is worthy calculating the precession and nutation amplitudes for a large set of asteroids; a method is explained in the following section.

\section{Precession and main nutation coefficients}

Here, we assume that each given asteroid considered in the following can be assimilated to a rigid body with a perfect ellipsoidal shape in which the moments of inertia $A, B, C$ satisfy $A<B<C$, with respect to the semi-axes $a, b, c$, where $a<b<c$. Moreover, we only take into account the effects of the Sun on the rotation, which is supposed to satisfy a short-axis mode, such that the rotation vector is very close to the figure axis that is of length $c$. For each asteroid, the expression of the disturbing potential exerted by the Sun in spherical harmonics limited at the first order is (Kinoshita (1977)):

$$
\begin{aligned}
U=\frac{\kappa^{2} M_{\mathrm{S}}}{r^{3}}\left(\left[\frac{2 C-A-B}{2}\right] P_{2}^{0}(\sin \delta)\right. \\
\left.+\left[\frac{A-B}{4}\right] P_{2}^{2}(\sin \delta) \cos 2 \alpha\right),
\end{aligned}
$$

where $\kappa^{2}$ is the gravitational constant, $M_{\mathrm{S}}$ is the mass of the Sun, and $r$ the heliocentric distance of the asteroid. $P_{2}^{0}(\sin \delta)$ is the associated Legendre polynomial and depends on the variable $\delta$, which represents the declination of the Sun with respect to the equator of the asteroid (containing the semi-axes with lengths $a$ and $b$ ). Here, the variable $\alpha$ does not designate the right ascension of the Sun but its longitude is counted from a prime meridian fixed to the asteroid. Therefore, the second term of the right hand side of Eq. (3) denotes high frequency with a cycle corresponding to half the rotational period of the asteroid due to the presence of the term $\cos 2 \alpha$. For this reason it can be shown (Souchay et al. 2003a) that this part of the potential does not participate to the precession and gives birth to very small nutation coefficients. Thus, we do not take it into account here.

In order to compute the precession rate and the leading nutation coefficient, we follow exactly the same procedure as Souchay et al. (2003b) when they precisely studied the rotation of the asteroid 433 Eros. For that purpose, they followed a complete analytical theory of the rotation of a rigid body in Hamiltonian formalism, which was developed by Kinoshita (1977), and applied to the Earth with a very high level of accuracy (Souchay et al. 1999).

Through the intermediary of Jacobi polynomials, the Legendre polynomial $P_{2}^{0}(\sin \delta)$ can be split into three components, involving the longitude of the Sun $\lambda_{\mathrm{S}}$, its latitude $\beta_{\mathrm{S}}$ that is defined with respect to an arbitrary reference plane and an arbitrary starting point, and $I$ which is the inclination of the asteroid equator that is defined with respect to this basic plane.

$$
\begin{aligned}
P_{2}^{0}(\sin \delta)= & \frac{1}{2}\left(3 \cos ^{2} J-1\right)\left[\frac{1}{2}\left(3 \cos ^{2} I-1\right) P_{2}^{0}\left(\sin \beta_{\mathrm{S}}\right)\right. \\
& -\frac{1}{2} \sin 2 I P_{2}^{1}\left(\sin \beta_{\mathrm{S}}\right) \sin \left(\lambda_{\mathrm{S}}-h\right) \\
& \left.-\frac{1}{4} \sin ^{2} I P_{2}^{2}\left(\sin \beta_{\mathrm{S}}\right) \cos 2\left(\lambda_{\mathrm{S}}-h\right)\right],
\end{aligned}
$$

where $J$ represents the angle between the directions of the angular momentum vector and of the figure axis. This angle is supposed to be very small in general, as it is for the Earth for which it does not exceed $1^{\prime \prime}$. It has never been detected for the other terrestrial planets, which supports the hypothesis that this smallness is also satisfied for them. Therefore, we can postulate that also for the asteroids investigated here. The variable $h$ is also a very small quantity, varying very slowly compared to $\lambda$, which represents the displacement of the starting point along the reference plane (Souchay et al. 2003b,a). It can be neglected if compared to $\lambda$ at first order. 
We have seen above that the basic plane from which obliquity, precession, and nutation are computed is arbitrary. A judicious choice is to take for this plane the orbital plane of the asteroid around the Sun, so that the latitude $\beta_{\mathrm{S}}$ is equal to zero. Thus,

$P_{2}^{0}(\sin \beta)=-\frac{1}{2}\left(1-3 \sin ^{2} \beta\right)=\frac{1}{2}$,

$P_{2}^{1}(\sin \beta)=3 \sin \beta \cos \beta=0$, and

$P_{2}^{2}(\sin \beta)=3 \cos ^{2} \beta=3$.

In that case, this leads to the final simplified formula for $U$ :

$U=k\left[\frac{a}{r}\right]^{3} \times\left[-\frac{1}{4}\left(3 \cos ^{2} I-1\right)-\frac{3}{4} \sin ^{2} I \cos \left(2 \lambda_{\mathrm{S}}-h\right)\right]$

with

$k=\frac{\kappa^{2} M_{\mathrm{S}}}{a^{3}}\left[\frac{2 C-A-B}{2}\right]$.

With the specific choice of the basic plane above, $I$ represents the obliquity: $I=\varepsilon$.

\subsection{Precession rate}

The precession rate $\dot{\psi}$ for any asteroid is calculated in a very straightforward manner by integrating the constant part of the potential in (8) (Kinoshita 1977; Souchay et al. 2003b). It is given by the following expression at the fourth order of the eccentricity:

$\dot{\psi}=\frac{K}{2} \times\left[1+\frac{3}{2} e^{2}+\frac{15}{4} e^{4}\right] \cos \varepsilon$,

where the expression in brackets, which depends on the sole eccentricity, corresponds to the constant part of the quantity $(a / r)^{3}$. The scaling factor $K$ is given at first order by:

$K=\frac{3 n^{2}}{\omega} \times H_{\mathrm{d}}=\frac{3 n^{2}}{\omega} \times\left(\frac{2 C-A-B}{2 C}\right)$,

where $H_{\mathrm{d}}=(2 C-A-B / 2 C)$ represents the dynamical ellipticity of the asteroid, which is characterized by its flattening. The parameter $n$ stands for the mean motion of the asteroid and $\omega$ for its angular rotational velocity.

\subsection{Nutation coefficients}

The nutation characterizes the small oscillations undergone by the pole of figure (or rotation) in space. It is obtained by keeping the solar potential periodic (sinusoidal) components. It is characterized by the oscillations of the obliquity $\Delta \varepsilon$ and of the longitude of the node $\Delta \psi$ between the equator of figure and the orbital plane of the asteroid. As expressed in a simplified manner by Souchay et al. (2003b,a), which starts from the formula in Kinoshita (1977), the nutations in longitude $\Delta \psi$ and in obliquity $\Delta \varepsilon$ can be approximated at first order by

$$
\begin{aligned}
\Delta \psi & =\frac{K \cos \varepsilon}{2} \times \int\left[\left[\frac{a}{r}^{3}\right]-\left[\frac{a}{r}^{3}\right] \cos \left(2 \lambda_{\mathrm{S}}-2 h\right) \mathrm{d} t\right]_{\mathrm{per} .}, \\
\Delta \varepsilon & =\frac{K}{2} \times \int\left[\left[\frac{a^{3}}{r}\right] \sin \left(2 \lambda_{\mathrm{S}}-2 h\right) \mathrm{d} t\right]_{\mathrm{per} .} .
\end{aligned}
$$

Here, the index per. means that we only keep the periodic (sinusoidal) part of the expression.
After integration, $\Delta \psi$ and $\Delta \varepsilon$ are given in the form of Fourier series with the same formalism as Souchay et al. (2003b) for Eros 433. By keeping only the leading components, we have

$$
\begin{aligned}
& \Delta \psi \approx \frac{K \cos \varepsilon}{2}\left[-\left(1-\frac{5}{2} e^{2}\right) \frac{\sin 2 \lambda}{2 \dot{\lambda}}+\frac{3 e \sin M}{\dot{M}}+\frac{e}{2} \frac{\sin \lambda}{\dot{\lambda}}+\cdots\right], \\
& \Delta \varepsilon \approx \frac{K \sin \varepsilon}{2}\left[\left(1-\frac{5}{2} e^{2}\right) \frac{\cos 2 \lambda}{2 \dot{\lambda}}-\frac{e}{2} \frac{\cos \lambda}{\dot{\lambda}}+\cdots\right],
\end{aligned}
$$

where $M$ is the mean anomaly of the asteroid, whereas $\lambda$ stands for its longitude, which is counted from the equinoctial point that is defined from the ascending node of the Sun relative orbital plane with respect to the asteroid equatorial plane. Because of the very slow motion of the asteroid equinoxes, the linear rates, $\dot{\lambda}$ and $\dot{M}$, can be considered as identical at first approximation. Therefore, the sinusoidal expressions with arguments of the form $\sin M$ and $\sin \lambda$ in Eq. (14) can be considered as having the same frequency with a phase shift that varyies very slowly with time.

We note that the period of the largest nutation oscillation in $\Delta \psi$ is either the orbital period of the asteroid (component with $\sin M$ ) or half this period (component with $\sin 2 \lambda$ ), according to the value of the eccentricity. In the case of $\Delta \varepsilon$ this indetermination does not exist for the term, where the argument $M$ does not exist. Therefore, the leading oscillation has always a semi-orbital period with argument $2 \lambda$.

\section{The available database}

To carry out our computations of obliquity, precession, and nutation, according to Eqs. (14) and (15), our study is based on the following four electronic databases:

(1) Planetary Data System Asteroid/Dust Archive ${ }^{1}$,

(2) Database of Asteroid Models from Inversion Techniques ${ }^{2}$,

(3) IAU Minor Planet Center ${ }^{3}$,

(4) Wolfram Curated Data (AstronomicalData) ${ }^{4}$.

The Planetary Data System (PDS) provides quick access to different kinds of data on small solar system bodies like planetary satellites, asteroids, comets, meteorites, and dust. For our study, the mass and major axis are taken from the Asteroid Masses V2.0, and the spin data is taken from the Asteroid Spin Vector Compilation V5.0 product (see webpage for more information).

The shape models of various asteroids and complementary information on the spin can be found in the Database of Asteroid Models from Inversion Techniques (DAMIT). The database not only contains well-curated data obtained from lightcurves of asteroids but also provides a compilation of additional asteroid data and relevant references. See the webpage of Durech et al. (2010) for more information.

The orbital elements are taken from the IAU Minor Planet Center (MPC), from the MPC Orbit (MPCORB) database. It contains the orbital elements of minor planets that have been published in the Minor Planet Circulars, the Minor Planet Orbit Supplement, and the Minor Planet Electronic Circulars.

Both data on rotational and orbital aspects of about 50000 asteroids can also be found in the AstronomicalData product of the Wolfram Curated Data (WCD; see webpage for

\footnotetext{
1 http://sbn.psi.edu/pds/

2 http://astro.troja.mff.cuni.cz/projects/asteroids3D/ web.php

3 http://www.minorplanetcenter.net/

4 Wolfram Mathematica or http://www . wolframalpha.com/
} 
further references). The data can be directly accessed through Wolfram Mathematica or through the Wolfram Alpha webpage. The database was used to cross-reference the orbital and rotational data if sufficient data are available. In the following, we refer (1)-(4) to the content of the databases taken from PDS, DAMIT, MPC and WCD, respectively.

\subsection{General information}

For the asteroids that were visited during space missions, data are taken from the web and from (1)-(4) (see also short discussion in Sect. 3.2). For all remaining asteroids the missing physical properties are taken from (1) and (2), and the orbital elements are taken from (3). All data are obtained in the following way: i) only objects, which are present in all four databases were taken into account. ii) the mass (if available) and radius of an individual object are obtained from (4), where the radius is cross-checked with the diameter published in (2). iii) the ratios of the semi axes $a / b$ and $b / c$ are taken from (1), and the numbers are cross-referenced with the ratios obtained from (2) by fitting an ellipsoid through the published shape models using a standard least squares method (see also Sect. 4). This also provides, in addition to $a / b$ and $b / c$, the axis $c$ in physical units. Note that depending on the numbers of shape models published for one object in (2), we may obtain more than one solution for $a / b, b / c$, and $c$ from the latter database. The rotation period of an individual object is taken from (2) and is cross-referenced with the rotation period of the same object published in (4). The spinpole position of an asteroid in ecliptic coordinates $(\lambda, \beta)$ is taken from (2) (given at epoch J2000); the values are cross-referenced with the spin-pole positions also published in (1). In the latter, the positions are given in terms of $(\lambda, \beta)$ but at epoch $B 1950$.

We note that up to four spin-pole positions may be found in (1) for one individual asteroid (the number depends on the method of determination of the spin-pole positions). In that specific case the positions come in two pairs of solutions (one value of one pair shifted by about $180^{\circ}$ from the second one). The orbital period is taken from (4), while the orbital elements are obtained from (3). There are cases, where all necessary data about the orbit, the spin vector and the geometry can be found in (1), but the asteroid is not listed in (2). The asteroids were added to our target list too. In total we were able to keep seven asteroids with data obtained from space missions, 34 asteroids with data from (1) and (2) and 59 asteroids with enough information from (1) but not from (2). Thus, we finally gathered 100 asteroids from which we have enough data to deduce the obliquity and precession periods.

We provide our target list (see Table 1) in which one data point entry consists of four lines. Each line starts with the IAU designation number of the asteroid:

\section{1:id., name,}

2:id., $m^{[1],[4]}, R^{[4]}[\mathrm{km}], a / b^{[1]}, b / c^{[1]}$, no., $c^{[2]}[k m], a / b^{[2]}, b / c^{[2]}$, 3:id., $T_{\text {rot }}^{[2],[4]}[h], \lambda^{[2]}, \beta^{[2]}, \varepsilon^{[2],[3]}$, no., $\lambda^{[1]}, \beta^{[1]}, \varepsilon^{[1],[3]}$, 4:id., $T_{r e v}^{[4]}[y], T 0^{[3]}, a^{[3]}[A U], e^{[3]}, i^{[3]}, \omega^{[3]}, \Omega^{[3]}, M^{[3]}, n^{[3]}\left[{ }^{\circ} / d\right]$.

In line $1 \mathrm{id}$. stands for the designation number, and name is the official IAU name of the object, as published in (3). In line 2: $m$, taken from (1) or (4), is the mass of the object given in the mass unit of Ceres; the equatorial radius $R$ is given in [km]; the first two ratios, $a / b$ and $b / c$, are the ratios of the semi axes published in (1); no. defines the number of shape models that exist for one asteroid in (2) from which $a, b, c$ and the respective ratios are calculated (see below).

In line $3, T_{\text {rot }}$ is the rotation period (in hours) of the asteroid as published in (2). The first three parameters $(\lambda, \beta, \varepsilon)$ denote the ecliptic longitude $\lambda$ and latitude $\beta$ as they are published in (2); the resulting obliquity $\varepsilon$ has been calculated on the basis of the orbital parameters (line 4). The integer no. gives the number of spin-vector solutions, which are published for one object in (1) (the number of triplets of the form $(\lambda, \beta, \varepsilon)$ that could be calculated using the different $(\lambda, \beta)$ that are published in (1) on the basis of the orbital parameters given in line 4$)$.

The first entry in line 4 is the orbital period in [y] published in (4), T0 defines the epoch for which the elements are given; $a$ is the semi-major axis in [AU]; $e$ is the eccentricity, $i$ is the inclination, $\omega$ is the argument of perihelium; $\Omega$ is the longitude of the ascending node; $M$ is the mean anomaly at $T 0$, and $n$ is the mean motion in $\left[{ }^{\circ}\right]$ and $[\%$ d]. We note that all values are taken as they are published in (1)-(4) with the exception of the second set of shape parameters $a / b, b / c$, and $c$ in line 2 , which were calculated from shape models published in (2) and the obliquities of the asteroids $\varepsilon$ in line3. The obliquities are obtained by combining the spin-vector solutions, which are published in (1) or (2) with the orbital parameters (published for the object in (3) and (4)) according to a method fully described in Sect. 5.

\subsection{Complementary information from space missions}

For some asteroids that have been visited during space missions, additional information is available: NASA's space probe Galileo (launched 1989) was the first to make a flyby near an asteroid (951 Gaspra) and discovered the first asteroid moon (Dactyl) around the asteroid 243 Ida. The NEAR Shoemaker mission (launched 1996) was designed to study the near-Earth asteroid 433 Eros in great detail over a period of a year and was the first probe to touch down on an asteroid surface (2001 Feb. 12). It also flew by the asteroid 253 Mathilde. The Deep Space 1 spacecraft (launched 1998), maintained by NASA, carried out a flyby of asteroid 9969 Braille and included an encounter with the comet, Borelly. For cost reasons, the asteroid 1999 KK1 was not visited by the spacecraft at the end of the mission.

The primary aim of the space mission named Stardust (launched 1999) was to collect dust samples from the coma of the comet Wild 2; but however it also flew by and studied the asteroid 5535 Annefrank. In 2000, the Cassini space probe passed the asteroid 2685 Masursky on its way to the planet Saturn. Another space mission called CONTOUR (launched 2002) investigated the nuclei of the two comets, Encke and Schwassmann-Wachmann-3, but did not visit an asteroid. The JAXA mission named Hayabusa, formerly known as MUSES-C (launched 2003), visited the asteroid 25143 Itokawa with the aim of returning the first sample of the surface of the asteroid back to Earth. The mission also included a lander (MINERVA), which unfortunately failed to reach the surface. An important contribution is from the European robotic spacecraft mission Rosetta (launched 2004), to study the comet 67P/ChuryumovGerasimenko in 2014. However, the space probe already completed its flybys of the asteroid 2867 Steins (2008), the asteroid P/2012 A2, and 21 Lutetia (2010). The space probe, Deep Impact (launched in 2005), visited the comet 9P/Tempel and will reach the asteroid (163249) 2002GT within the year 2020. The asteroid 4 Vesta was already visited by NASA's DAWN spacecraft during 2011,2012 with the aim to reach the dwarf planet Ceres in 2015. 
Future missions with possible flybys of asteroids or minor planets include: the NASA mission New Horizons, which already had its closest approach with the asteroid 132524 APL in the year 2006; the Chinese space probe Chang'e 2, which will visit the asteroid 4179 Toutatis; the JAXA mission Hayabusa 2 (targeting the asteroid $1621731999 \mathrm{JU}_{3}$ with a lander called MASCOT, which is going to be developed at the DLR in collaboration with the French space agency CNES; Don Quijote, which is a proposed space probe by the European Space Agency to investigate the effects of crashes into an asteroid (2003 SM 84 or 99942 Apophis); the US space mission OSIRIS-REx, which aims to return a sample from the asteroid $1999 \mathrm{RQ}_{36}$; the ESA mission AIDA with possible target 65803 Didymos; and the MarcoPolo-R mission, which in the framework of the Cosmic Vision program is supposed to return a sample from the binary asteroid system (175706) $1996 \mathrm{FG}_{3}$. In total, we found 14 asteroids, which were visited during space missions in the last 30 years. We could collect enough data for only seven of them for the purpose of the present calculations.

\section{Computation of the moments of inertia}

For any given asteroid, the principal moments of inertia $A<B<$ $C$ are linked to the second degree spherical harmonics by

$J_{2} M R^{2}=C-(A+B) / 2, \quad c_{22} M R^{2}=(B-A) / 4$,

where $M$ and $R$ are the mass and radius of the asteroids and where the coefficients $J_{2}$ and $c_{22}$ can be obtained from experiments in space. However, only a few asteroids have been visited by space probes so far, and we are able to determine the gravity field for only a few of them. As a consequence, $J_{2}$ and $c_{22}$ are unknown for the majority of the asteroids, and we cannot use them to calculate $A, B$ and $C$ directly. As a first approximation, we assume that the asteroid is of uniform density and can be approximated by an ellipsoidal shape. In that case, the principal moments of inertia are given by Bills \& Nimmo (2011):

$A=\frac{1}{5} M\left(b^{2}+c^{2}\right), B=\frac{1}{5} M\left(a^{2}+c^{2}\right), C=\frac{1}{5} M\left(a^{2}+b^{2}\right)$.

Here $a, b$ and $c$ are the semi axes of the fitting ellipsoid, and $M$ is the mass of the asteroid, which is also not known with enough accuracy for the large majority of asteroids. Nevertheless we can have access to the ratios, $a / b$ and $b / c$, which can be estimated directly from observations (from the shape of the asteroid itself obtained from lightcurve inversion techniques Kaasalainen \& Torppa 2001; Kaasalainen et al. 2001).

In (2), different shape models are given for an individual asteroid in the form of polyhedrons with triangular surface facets in terms of vertex coordinates $\left(x_{i}, y_{i}, z_{i}\right) \in \mathbb{R}^{3}$ with $i=1, \ldots, N \in \mathbb{N}$ and order numbers $\left(\alpha_{j}, \beta_{j} \gamma_{j}\right) \in \mathbb{Z}^{3}$ for each facet with $j=1, \ldots, M \in \mathbb{N}$. For our purpose, only the vertex coordinates are used: the data points fit an ellipsoid with free parameters $a, b$ and $c$ through them by making use of a standard least squares method. We thus aim to minimize the quantity,

$d_{i}(a, b, c)=\frac{x_{i}^{2}}{a^{2}}+\frac{y_{i}^{2}}{b^{2}}+\frac{z_{i}^{2}}{c^{2}}-1$,

with respect to the unknown parameters $(a, b, c)$, where $i=$ $1, \ldots, N$. The typical number of points $N$ used in the fitting process are on the order of $10^{2}$ to $10^{3}$. Moreover, the typical absolute errors (absolute difference of $d_{i}$ from zero) ranges from a fraction of 1 percent to 10 percent of the mean radius. We note that the final values $(a, b, c)$ in a few cases are switched to follow the usual convention $a \geq b \geq c$. We indicate this by a $*$ and + in Table 2.

In our ongoing calculations, we only need the dynamical ellipticity $H_{\mathrm{d}}$, which is given by

$H_{\mathrm{d}}=\frac{2 C-A-B}{2 C}=\frac{1}{2}-\left[\left(\left(\frac{a}{b}\right)^{2}+1\right)\left(\frac{b}{c}\right)^{2}\right]^{-1}$,

where the right hand-side comes from the contribution of (17) combined with the middle of (19). As we can see, $H_{\mathrm{d}}$ has become independent of the mass $M$, and only the ratios $a / b$ and $b / c$ take part to its calculation. We are thus able to take into account all the asteroids for which only the relative ratios of the semi-axes are known, even if the masses are usually not. To test the validity of our approach we also use the algorithm described in Mirtich (1996) to directly calculate the principal moments of inertia matrix from the shape models that are published in (2). Let us denote $e_{x}, e_{y}, e_{z}$ the eigenvectors, and $\lambda_{x}, \lambda_{y}, \lambda_{z}$ the coresponding eigenvalues of the moments of inertia matrix. From the formulae, $2 / 5 m a^{2}=\lambda_{y}+\lambda_{z}-\lambda_{x}$ and $2 / 5 m b^{2}=\lambda_{z}+\lambda_{x}-\lambda_{y}$, $2 / 5 m c^{2}=\lambda_{x}+\lambda_{y}-\lambda_{z}$, we are able to obtain the ratios $a / b$ and $b / c$, without fitting an ellipsoid to the data set.

\section{Determination of obliquities}

The obliquity of each asteroid is a fundamental parameter for our calculations. We calculate the value of the obliquity $\varepsilon$ from the knowledge of the spin and orbit poles of the asteroid. First, the direction of the spin pole, $f$, is defined in terms of its ecliptic longitude $\lambda$ and latitude $\beta$ by

$\boldsymbol{f}=\left(\begin{array}{c}\cos \beta \cos \lambda \\ \cos \beta \sin \lambda \\ \sin \beta\end{array}\right)$

Second, the unit vector with a direction parallel to the orbit pole, $\boldsymbol{o}$, which is normal to the asteroid's orbit, is given by

$\boldsymbol{o}=\left(\begin{array}{c}\sin i \sin \Omega \\ -\cos \Omega \sin i \\ \cos i\end{array}\right)$.

The obliquity $\varepsilon$ can be obtained by inverting the two following formulae:

$\boldsymbol{o} \cdot \boldsymbol{f}=\cos \varepsilon, \boldsymbol{o} \times \boldsymbol{f}=\boldsymbol{w} \sin \varepsilon$,

where $\boldsymbol{w}$ is the unit vector along the direction of the descending node of the asteroid's orbit with respect to the true equator. In $(1),(\lambda, \beta)$ are given with respect to $\mathrm{J} 2000$; in (2), they are given with respect to B1950. To transform from B1950 to J2000, we simply add $0.7^{\circ}$ to the ecliptic longitude $\lambda$, which is accurate enough, since the accuracy of the spin pole positions is typically about a few degrees.

If the spin pole is given in the equatorial coordinate system in terms of the declination $\delta$ and right ascension $\alpha$, these coordinates can be easily transformed to the ecliptic system by (Bills \& Nimmo (2011))

$\beta=\sin ^{-1} q_{3}, \lambda=\tan ^{-1} \frac{q_{2}}{q_{1}}$.

If we define the rotation matrix $R_{1}=R_{1}(\varphi)$ about the angle $\varphi$ as

$R_{1}=\left(\begin{array}{ccc}1 & 0 & 0 \\ 0 & \cos \varphi & -\sin \varphi \\ 0 & \sin \varphi & \cos \varphi\end{array}\right)$, 

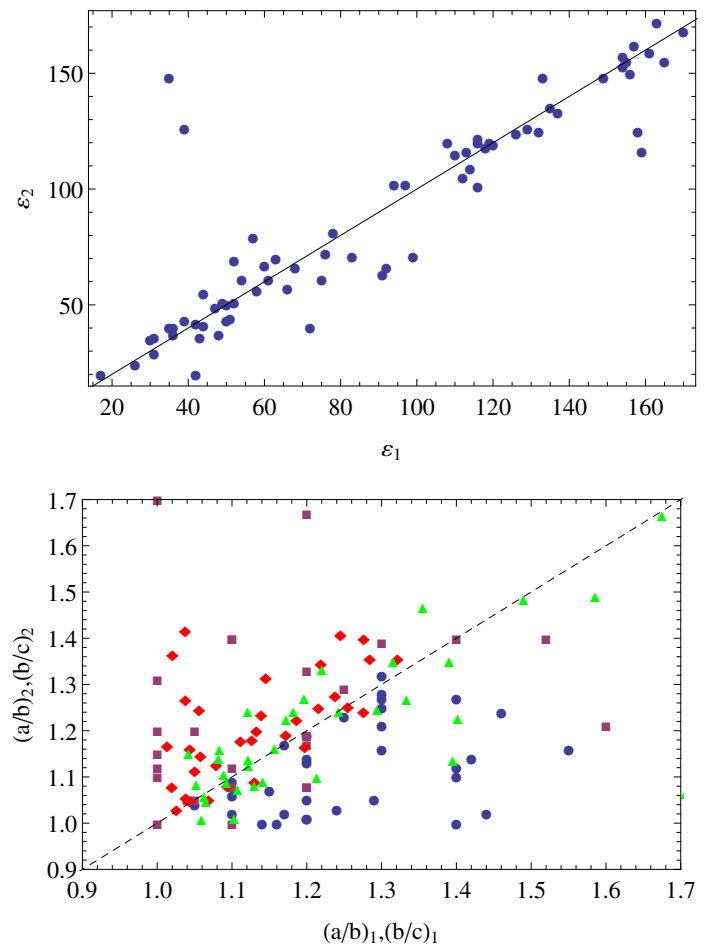

Fig. 1. Pairs of obliquities, $\left(\varepsilon_{1}, \varepsilon_{2}\right)$, found for the same asteroid but taken from different data sources (top), pairs of shape parameters, $\left((a / b)_{1},(a / b)_{2}\right)$ and $(b / c)_{1},(b / c)_{2}$, for the same asteroid but from different data sources (bottom). The plot markers indicate the different source of the data, see text.

the $\left(q_{1}, q_{2}, q_{3}\right)$ can be calculated from

$\left(\begin{array}{l}q_{1} \\ q_{2} \\ q_{3}\end{array}\right)=R_{1}\left(-\varepsilon_{\mathrm{E}}\right)\left(\begin{array}{c}\cos \delta \cos \alpha \\ \cos \delta \sin \alpha \\ \sin \delta\end{array}\right)$,

where $\varepsilon_{\mathrm{E}}$ stands for the obliquity of the Earth. That is $\varepsilon_{\mathrm{E}}=$ $23^{\circ} 26^{\prime} 21.448^{\prime \prime}$ (J2000).

\section{Presentation of basic tables}

In this section we collect the main results of our study, consisting of 100 asteroids for which we were able to calculate the obliquity, precession rate, and nutation coefficients (Table 2).

The quality of our data is mainly limited by the accuracy of the observations and measurements at the present day. Out of hundreds of thousands of known asteroids, we only collected data from 100 asteroids for which the dynamical ellipticity and the obliquity, which are crucial in the calculation of the precession rates and nutation coefficients, could be determined with enough accuracy.

Moreover, the published values for $a / b$ and $b / c$ are determined with known errors and oversimplified assumptions, such as the assumption that the shapes of asteroids are ellipsoids. As a result, our results strongly depend on the method that the authors used to determine them and as a consequence, different values exist for the same object.

To get an idea of the widespread values of obliquities, we plot two estimations of them against each other in the upper plot of Fig. 1. In other words, we have pairs of obliquities $\left(\varepsilon_{1}, \varepsilon_{2}\right)$, of the same asteroid taken from two different databases. The values range from $17^{\circ}$ to $172^{\circ}$. With the exception of a few outliers, we can remark that the values, which we have calculated in both

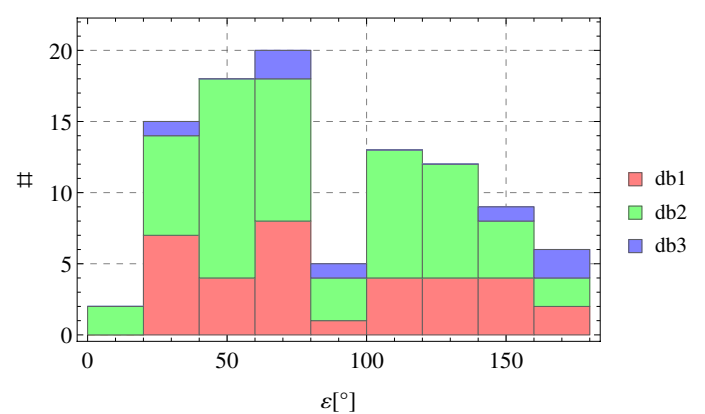

Fig. 2. Distribution of obliquities for 100 asteroids for different subsets $d b 1, d b 2, d b 3$ and all of them.

cases from the published values of the spin- and orbit-axes directions with Eq. (22) are in good agreement.

The situation is different when we compare the ratios $a / b$ and $b / c$ for the asteroids that are common in the two different databases, that were obtained from the two different shape models, or that were determined through the two different methods, as described in Sect. 4. Indeed, we find more than one solution for the ratios of 34 asteroids among our set of 100 objects (see Fig. 1): similar to the pairs of obliquites $\left(\varepsilon_{1}, \varepsilon_{2}\right)$, we found different pairs of ratios $(a / b)_{1},(a / b)_{2}$ and $(b / c)_{1},(b / c)_{2}$ for the same asteroid that were taken from two different data sources. This is due to the different shape models and different determination of these ratios. The deviation of the filled disks (squares) from the median indicates the difference of the ratios $a / b$ and $b / c$ originating from different shape models; filled diamonds (triangles) reveal the difference of the ratios $a / b(b / c)$ due to the two determination methods. We clearly see that the uncertainty in the shape models is about the order of magnitude of the error that we introduced when fitting just an ellipsoid. It is thus safe to use the first method, as described in Sect. 4 to obtain the values $a / b$ and $b / c$ for the ongoing calculations. The uncertainty in these ratios directly affects the uncertainty in the dynamical ellipticity $H_{\mathrm{d}}$. We will address the issue one more time in the following section (Fig. 4).

\section{Results and statistics}

In the following discussion we split our dataset into three different sets:

- $d b 1$ contains the data of the asteroids for which it was possible to find information for the shape and for the spin- and orbit-axes from which we calculated the obliquity in both (1) and (2).

- $d b 2$ contains the data for which this same information (enabling the deduction of $\varepsilon, a / b$ and $b / c$ ) can only be found in (2) but not in (1).

- $d b 3$ concerns the asteroids for which (generally accurate) data are taken from space missions.

In $d b 1$, we gather 34 asteroids (arranged in Table 1 in chronological order from 2 Pallas to 276 Adelheid); in $d b 2$, we have 59 asteroids (from 1 Ceres to 77 Frigga). In $d b 3$, we have only 7 minor planets (from 951 Gaspra to 4 Vesta). From the three databases presented above, we are able to perform some basic descriptive statistics based on either an individual database or the full set of data which is the combination of $d b 1, d b 2, d b 3$. They are illustrated by the following figures.

The results on the obliquites of the asteroids of our solar system are shown in Fig. 2: the obliquites range from $9^{\circ} \leq \varepsilon \leq 170^{\circ}$ 
with a median value of about $75^{\circ}$. On the basis of our data, we find significantly less asteroids within the interval $-20^{\circ}$ (or $160^{\circ}$ ) to $20^{\circ}$. We remark that no asteroid was found between $-8^{\circ}\left(172^{\circ}\right)$ to $8^{\circ}$. Moreover, we also find a relative deficiency of asteroids with large obliquity which means that their polar axis is close to the orbit (and generally to the ecliptic). Only eight objects (2, Pallas, 7 Iris, 8 Flora, 16 Psyche, 22 Kalliope, 28 Bellona, 433 Eros, and 704 Interamnia) are found in the range $80^{\circ}<\varepsilon<100^{\circ}$. This conforms the remark made by Michalowski (1993) and also agrees with Magnusson (1986, 1990) who analyzes the spin vectors of 20 to 30 asteroids respectively. When quoting the apparent lack of poles with axes close to the ecliptic, this last author attributed this deficiency to an observational selection effect: an asteroid with a pole at a low ecliptic latitude is naturally less suitable to present a significant amplitude of the lightcurve variation. He concluded that these asteroids needed more lightcurve observations than for an asteroid with a polar axis close to the ecliptic. Drummond et al. $(1988,1991)$ confirmed the bimodality of the observed pole distribution using results for 26 asteroids. If they used the same explanation as Magnusson $(1986,1990)$ for this distribution, they did not exclude the possibility that the bimodality may be real and may reflect a primordial distribution of spin rates. Another confirmation of the non-uniform distribution of spin axes comes from Pravec et al. (2002), who use a larger sample of asteroid-pole estimates than the authors above. Their dataset comes mainly from two databases, the NASA Planetary Data System and the Uppsala's website, which is completed by other studies. Their sample with a total of 83 asteroids showed once more that the distribution of obliquities is bimodal and not flat. They carried out a Kolmogorov-Smirnov test, showing that the distribution is not uniform at a $85 \%$ confidence level.

Another factor of study concerns the relative amount of prograde/retrograde rotations. If we neglect $d b 3$, which is not statistically representative (for it contains only 7 asteroids), for each of the samples considered in our study, the number of asteroids having $\varepsilon<90^{\circ}$ (prograde case) is significantly larger than the opposite (retrograde case), as can be seen in Fig. 2. For the whole sample, we have 57 prograde objects against 43 retrograde ones. These values that translate into percentages (using the complete set of 100 asteroids) are in very good agreement with those found by Pravec et al. (2002), who found 48 prograde rotations versus 32 retrograde ones. These values correspond respectively to $60 \%$ and $40 \%$ of their sample.

Moreover, it is worthy to mention an important point: all the authors quoted above in this section consider the obliquity of the spin axis with respect to the ecliptic, whereas we calculate the true obliquity, which is characterized by the angle between the spin axis and the orbital one in this study. The difference between the values deduced from the two methods could reach (in the optimal case) the value of the inclination of the orbit of the asteroid with respect to the ecliptic, which corresponds to a substantial amount (more, and sometimes much more than $10^{\circ}$ ) in some cases. Therefore, the statistics of these authors should be a little affected by their approximate method of calculation. Nevertheless, it is true that the corresponding error should be compensated by the uncertainty concerning the spin axis orientation itself, which is generally determined with error bars of a few degrees.

In Fig. 3, we report the distribution of the shape parameters $a / b$ and $b / c$. We can remark in particular that values of the ratios $a / b$ and $b / c$ are significantly more probable to be found close to 1 than values far from unity. The median values for these ratios are both close to 1.2. However, one should notice that the
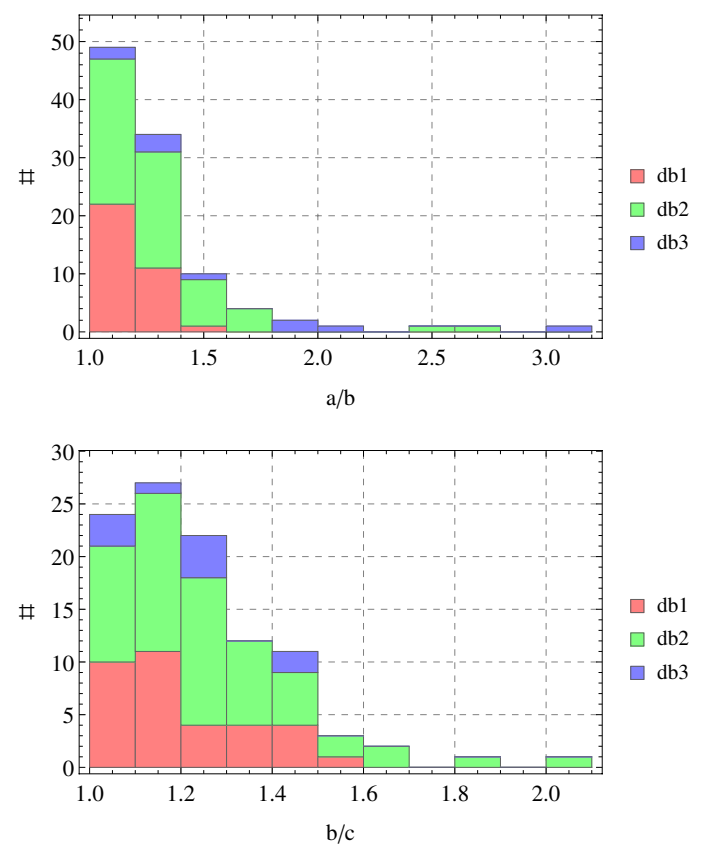

Fig. 3. Distribution of $a / b$ (top) and $b / c$ (bottom) for 100 asteroids for the different subsets $(d b 1, d b 2, d b 3)$ and for all of them.
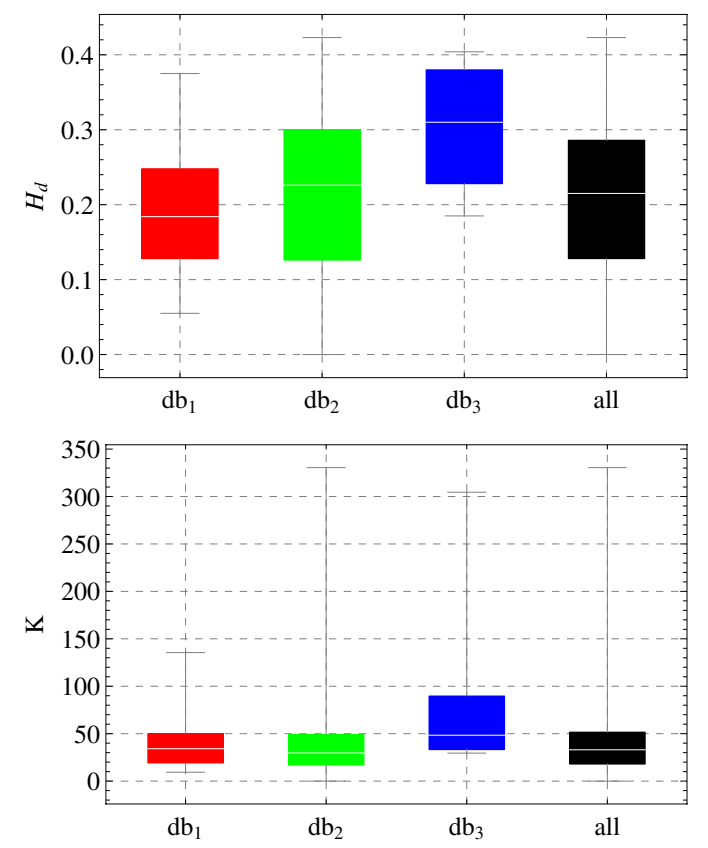

Fig. 4. Distribution of $H_{\mathrm{d}}$ (top) and $K$ (bottom) for 100 asteroids for the different subsets $(d b 1, d b 2, d b 3)$ and for all of them. The filled rectangle marks the $75 \%$ quantile. Lines bound the upper and lower range, the center line marks the median value of the respective data set.

distribution is biased because the shape parameters cannot be derived easily and usually exhibit large error bars (see bottom of Fig. 1).

The distributions of the dynamical ellipticity $H_{\mathrm{d}}$ and of the scaling factor $K$, which plays a direct and fundamental role in the amplitude of the precession rate and nutation coefficients, are shown in Fig. 4. The median value of $H_{\mathrm{d}}$ in $d b 1$ lies close to 0.184 and increases to 0.226 for $d b 2$ and 0.310 for $d b 3$. In the global data set, we find a median value of about 0.215 . The values seem to be randomly distributed with large variations (between 0 and 0.423 , as seen in the error bars), with a confidence 
interval between 0.128 and 0.286 . Some statistics on the scaling factor $K$ are shown in Fig. 4 (bottom), ranging from 0 to $330^{\prime \prime} / y\left(881^{\prime \prime} / y\right.$ for the outlier 25143 Itokawa) with a median value of about $33^{\prime \prime} / y$. We notice that the dynamical ellipticity for the Earth, which is rather poorly flattened with respect to the objects studied here, is $H_{\mathrm{d}}^{\text {Earth }} \approx 0.0033$, and the scaling factor $K^{\text {Earth }}$ for the Sun part of the potential (our sole interest here) is $K^{\text {Earth }}=34.38^{\prime \prime} / y$. Therefore, the values of $H_{\mathrm{d}}$ are typically larger by one or two orders than for our planet, as we can see from Table 1 or Table 2, but the values of $K$ are of the same order.

In Fig. 5 we collect the results on the precession rate (top figure) and the leading nutation coefficients. We remark that we adopt the same conventions for the sign of the precession rate as for the Earth. This means that a positive sign characterizes a retrograde motion of the equinox. Moreover, this sign depends only on the value of the obliquity with respect to $90^{\circ}$, as can be checked in Eq. (8). As a consequence of the distribution of the obliquities discussed above, most of the asteroids are found to have $\dot{\psi}$ positive for our four data sets. As we can deduce from Table 2, the mean absolute value of this rate is $18.07^{\prime \prime} / y$, which can be compared with the solar part of the precession of the Earth $\left(15^{\prime \prime} / y\right)$ and the combined lunisolar part $\left(\approx 50^{\prime \prime} / y\right)$. In most of the cases here, the absolute value of the precession rate has smaller amplitudes than this last value, with the exception of 25143 Itokawa $\left(-475^{\prime \prime} 7 / y\right), 624$ Hektor $\left(-677^{\prime} \cdot 3 / y\right), 60$ Echo $\left(90{ }^{\prime} 5 / y\right), 277$ Elvira $\left(-677^{\prime} 47 / y\right)$, and 71 Niobe $(52$ '. $2 / y)$.

The absolute values of the leading nutation coefficients in longitude $\Delta \psi_{1}$ for the semi-annual oscillation (half the orbital period) and $\Delta \psi_{2}$ for the annual one are given simultaneously for all the objects of our sample. For $\Delta \psi_{1}$, the distributions deduced from Table 2 are shown in Fig. 5 (upper middle). They range between $0^{\prime \prime}$ and $41^{\prime \prime}$ (for 23145 Itokawa) with a mean value of about 4 .'5. The distribution is quite similar for $\Delta \psi_{2}$ (see lower middle of Fig. 5) with a minimum value at $0^{\prime \prime}$, a maximum value around 93" (still for 23145 Itokawa), and a mean value of about 4.'5, which is close to the case of $\Delta \psi_{1}$. The mean absolute value of $\Delta \psi$ (taken as the sup of $\Delta \psi_{1}$ and $\left.\Delta \psi_{2}\right)$ is $5^{\prime \prime} .72$.

Our final study concerns the nutation coefficient $\Delta \varepsilon$ in Fig. 5 (bottom): we find $0^{\prime \prime} \leq \Delta \varepsilon \leq 30$ '.21, with a median value of $3^{\prime \prime}$.53 and a mean value of 5'.17. To compare with the Earth, the leading nutation due to the sole solar gravitational effect is semi-annual with absolute amplitudes - roughly $1^{\prime \prime}$ for $\Delta \psi$ and 0.5 for $\Delta \varepsilon$. Therefore, the solar nutation amplitudes of the asteroids are generally significantly larger than for our planet.

\section{Conclusion}

In the present study, we calculated a complete set of the obliquities, precession rates and nutation coefficients for a sample of 100 (out of about 550000 known) asteroids for the first time. For our calculations we extracted the relevant data from four main databases for which the shape parameters $a / b$ and $b / c$ are known (or can be calculated from shape models) to be able to determine the dynamical ellipticity $H_{\mathrm{d}}$ and the scaling parameter $K$. We selected only the asteroids for which the spin-pole position is well determined, to calculate the obliquity $\varepsilon$ that is needed to deduce the precession rate $\dot{\psi}$ and the leading nutation coefficients, $\Delta \psi$ and $\Delta \varepsilon$. Our calculations were based on the theory of the rotational motion of a rigid body developed by Kinoshita (1977) and have already been applied for the asteroid Eros 433 by Souchay et al. (2003b,a). We present all our results in form of a database of 100 asteroids in Table 2. The relevant data to construct this table is presented in Table 1 .
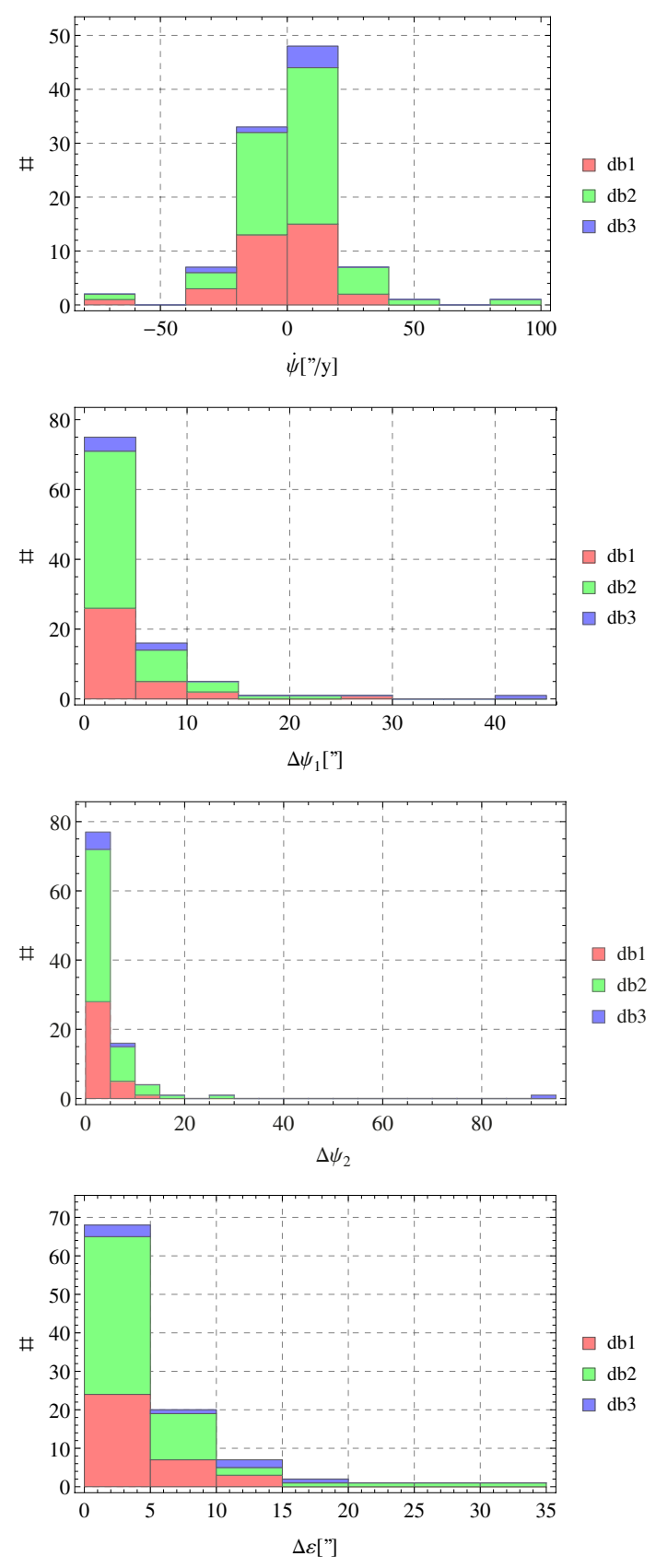

Fig. 5. Distribution of $\dot{\psi}$ (top), $\Delta \psi_{1}$ (upper middle), $\Delta \psi_{2}$ (lower middle) and $\Delta \varepsilon$ (bottom) for 100 asteroids for the different subsets $(d b 1, d b 2$, $d b 3)$ and for all of them.

Thanks to our relatively large sample of 100 asteroids, we were able to analyze the asteroid data and to derive some statistical properties of the rotational characteristics. Concerning the distribution of obliquities, main features are revealed, which agree with previous authors (Drummond et al. (1988, 1991); Magnusson (1986, 1990); Michalowski (1993); Pravec et al. (2002)). However, the results in these studies are affected, because they aproximated the orbital plane by the ecliptic for the calculation of the obliquity, while we used the true orbital plane that enabled us to calculate the more accurate value of the obliquity.

In our study, the obliquities of the asteroids are spread over the interval $9^{\circ} \leq \varepsilon \leq 170^{\circ}$ with the most representative value of about $75^{\circ}$, which shows that objects in the prograde rotation regime are significantly more numerous than those in the 
retrograde one. The mean precession rate $\dot{\psi}$ of the asteroids was found to be $2.87^{\prime \prime} / y$, and its mean absolute value is $18^{\prime \prime} 07 / y$, which is comparable to the amplitude of the solar component precession of the Earth $\left(15^{\prime \prime} / y\right)$. The nutation coefficients are typically on the order of a few arcseconds; the mean absolute value for the leading term are 5".72 for $\Delta \psi$ and 5'. 18 for $\Delta \varepsilon$. All these values can reach significantly larger values for some specific objects, such as for as 60 Echo, 71 Niobe, 624 Hektor, and 277 Elvira. The largest values are obtained for 25143 Itokawa with $\dot{\psi}=-475.7^{\prime \prime} / y$ and $\Delta \psi=93^{\prime \prime} 3$. Corresponding to a precession cone described in only 2725 years, this supposes a very fast precession motion, which may be detectable from lightcurves within two or three decades.

In conclusion, this paper constitutes an interesting work to better understand the evolution of the rotational motion of the asteroids. In particular, it presents a statistical analysis of the precession rates and nutations of the asteroids for the first time. It extends statistical studies to a larger set of objects, to computations of the obliquity variations, and to the precession-nutation effects.

\section{References}

Bills, B. G., \& Nimmo, F. 2011, Icarus, 213, 496

Burns, J. A., \& Safronov, V. S. 1973, MNRAS, 165, 403

Drummond, J. D., Weidenschilling, S. J., Chapman, C. R., \& Davis, D. R. 1988, Icarus, 76, 19

Drummond, J. D., Weidenschilling, S. J., Chapman, C. R., \& Davis, D. R. 1991, Icarus, 89, 44

Durech, J., Sidorin, V., \& Kaasalainen, M. 2010, A\&A, 513, A46

Harris, A. W. 1994, Icarus, 107, 209

Kaasalainen, M., \& Torppa, J. 2001, Icarus, 153, 24

Kaasalainen, M., Torppa, J., \& Muinonen, K. 2001, Icarus, 153, 37

Kinoshita, H. 1977, Celest. Mech., 15, 277

Kinoshita, H. 1992, Celest. Mech. Dyn. Astron., 53, 365

Magnusson, P. 1986, Icarus, 68, 1

Magnusson, P. 1990, Icarus, 85, 229

Michalowski, T. 1993, Icarus, 106, 563

Mirtich, B. 1996, J. Graph. Tools, 31,

Pravec, P., Harris, A. W., \& Michalowski, T. 2002, Asteroids III, 113

Rambaux, N., Castillo-Rogez, J., Dehant, V., \& Kuchynka, P. 2011, A\&A, 535, A43

Souchay, J., Loysel, B., Kinoshita, H., \& Folgueira, M. 1999, A\&AS, 135, 111

Souchay, J., Folgueira, M., \& Bouquillon, S. 2003a, EMP, 93, 107

Souchay, J., Kinoshita, H., Nakai, H., \& Roux, S. 2003b, Icarus, 166, 285 